\title{
Plasma processing of large curved surfaces for superconducting rf cavity modification
}

\author{
J. Upadhyay, ${ }^{1}$ Do Im, ${ }^{1}$ S. Popović, ${ }^{1}$ A.-M. Valente-Feliciano, ${ }^{2}$ L. Phillips, ${ }^{2}$ and L. Vušković ${ }^{1}$ \\ ${ }^{1}$ Department of Physics-Center for Accelerator Science, Old Dominion University, \\ Norfolk, Virginia 23529, USA \\ ${ }^{2}$ Thomas Jefferson National Accelerator Facility, Newport News, Virginia 23606, USA
}

(Received 26 June 2014; published 15 December 2014)

\begin{abstract}
Plasma-based surface modification of niobium is a promising alternative to wet etching of superconducting radio frequency (SRF) cavities. We have demonstrated surface layer removal in an asymmetric nonplanar geometry, using a simple cylindrical cavity. The etching rate is highly correlated with the shape of the inner electrode, radio-frequency (rf) circuit elements, gas pressure, rf power, chlorine concentration in the $\mathrm{Cl}_{2} / \mathrm{Ar}$ gas mixtures, residence time of reactive species, and temperature of the cavity. Using variable radius cylindrical electrodes, large-surface ring-shaped samples, and dc bias in the external circuit, we have measured substantial average etching rates and outlined the possibility of optimizing plasma properties with respect to maximum surface processing effect.
\end{abstract}

DOI: 10.1103/PhysRevSTAB.17.122001

PACS numbers: 81.65.Cf, 52.77.Bn, 74.70.Ad

\section{INTRODUCTION}

To improve the rf performance of superconducting radio frequency (SRF) niobium cavities, the cavity surface must be prepared by a process that enhances surface smoothness, removes impurities, and creates less sharp grain boundaries. Buffered chemical polishing or electropolishing [1] are currently used technologies based on the use of hydrogen fluoride (HF) in liquid acid baths, which poses major environmental and personal safety concerns. HF-free plasma-based ("dry") technologies are much more controllable, less expensive, and more environment friendly. To the best of our knowledge, we present the first results for three-dimensional plasma etching of the inner surface of bulk niobium cavities.

As a proof of concept, we developed an experimental setup for etching small flat niobium samples [2,3]. Microwave plasma at $2.45 \mathrm{GHz}$, inside a quartz tube, was used for this experiment. The gas mixture consisted up to $3 \%$ chlorine diluted in argon. The results were very encouraging [3], with etching rates up to $1.7 \mu \mathrm{m} / \mathrm{min}$. In every case, the surface roughness of plasma etched samples was equal or lower than the chemically etched samples.

The action in the development of plasma etching technology for SRF cavities is to remove niobium from inner surface of a cylindrical cavity. This makes the transition from small flat coupons to large curved surfaces. It also transitions from symmetric to asymmetric discharges in the coaxial electrode arrangement, since the inner and the

Published by the American Physical Society under the terms of the Creative Commons Attribution 3.0 License. Further distribution of this work must maintain attribution to the author $(s)$ and the published article's title, journal citation, and DOI. outer electrodes do not have the same surface area. We have achieved plasma etching by a cylindrical coaxial capacitively coupled rf discharge [4], which properties are described in the following section. The actual experimental setup for present work is described in the Sec. III, and the experimental results are discussed in Sec. IV.

\section{COAXIAL CAPACITIVELY COUPLED RF PLASMA}

In the experiments described here a coaxial plasma is generated using an electrode running coaxially with the material cylinder to be etched. The plasma is excited with a rf wave form of $13.56 \mathrm{MHz}$. This frequency was chosen because its wavelength is much larger than the characteristic size of the sample being etched and is expected to give more uniform results.

The plasma produced is in a capacitively coupled plasma (CCP) regime. In CCP when one electrode has a smaller area than the other, to maintain current continuity, the smaller area electrode acquires a negative dc voltage (self-bias [5,6]) as shown in Fig. 1.

Properties of asymmetric discharges were extensively studied in the development of planar semiconductor processing technology, starting as early as the 1970s [6,7]. Koenig and Maissel [7] used simple arguments, such as constant ion density near both electrodes, Child's law, and absence of collisions in the sheaths, to conclude that the inverse scaling of an electrode's voltage and surface area follows a power law with a scaling exponent of 4 . A number of later experimental works implied a much lower exponent [8-11]. We are presently interested in the cylindrical geometry where some experiments and models do exist $[10,12]$. 


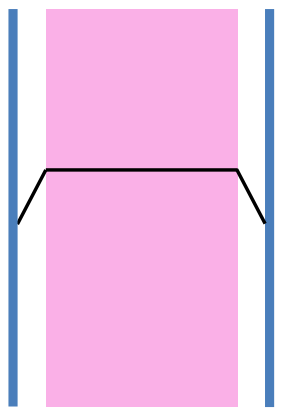

Symmetric discharge

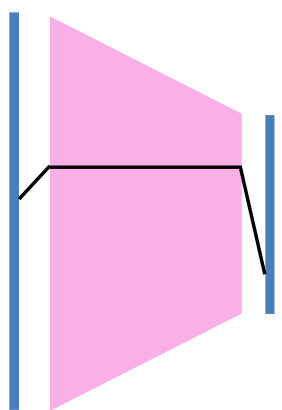

Asymmetric discharge
FIG. 1. Plasma sheath potential distribution in symmetric and asymmetric plasma.

The shape of a SRF cavity presents a challenge for $\mathrm{rf}$ plasma processing of its inner wall. It has a curved cylindrical symmetry and therefore, the processed surface has a larger area than the surface of the inner electrode. By contrast, the mature plasma processing technology, such as semiconductor wafer processing, is based on planar geometry. Moreover, the wafer to be etched is placed on the smaller-area electrode in order to take advantage of the asymmetry in the plasma sheath voltage. In the present case, the cavity that has to be etched is grounded and has a large surface area, so, in the absence of positive dc voltage on the inner electrode, the sheath potential is substantially lower on the cavity surface than the inner electrode.

Although we produce chemical radicals (excited neutral atom, molecule, and ions) of chlorine by the plasma in order to carry out the required reactions for material removal from the niobium surface, we need a potential for the ions to be accelerated prior to hitting the surface. Therefore, grounded niobium surface etching requires bringing the driven (inner) electrode to a positive dc potential with rf power. Details about the self-bias potential and the dependence of each rate on other parameters describing the etch mechanism will be published in a separate paper.

\section{EXPERIMENTAL SETUP}

We constructed a simple cylindrical cavity of $7.2 \mathrm{~cm}$ internal diameter and $15 \mathrm{~cm}$ length with 8 mini conflat ports. Some ports held niobium samples and others had a view port for diagnostic purposes. The cavity is shown in the Fig. 2.

The main goal of this preliminary experiment was to examine the etching on the outer electrode surface. For this purpose we opted for a variable diameter of inner electrode with the aim to observe the influence of the surface area of the inner electrode on the plasma properties. A set of variable diameter electrodes with the cylindrical cavity is shown in Fig. 3.

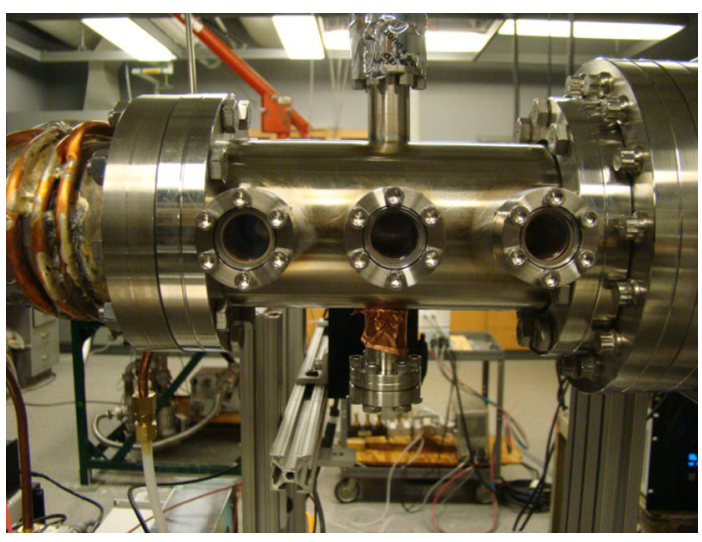

FIG. 2. Cylindrical processing cavity.

The experimental setup for the cylindrical cavity plasma processing is shown in Fig. 4. The cylindrical chamber is evacuated with roughing and turbo vacuum pumps. The gas flow and rf power are applied in the opposite direction, as shown in Fig. 4. The red arrow is in the gas flow direction and the blue arrow is in the power flow direction. The gas is mixed in a three-branch manifold, each branch containing mass flow meters. The first gas line is attached to the premixed gas cylinder of $15 \% \mathrm{Cl}_{2}$ diluted in $\mathrm{Ar}$ and the second gas line is connected to the pure Ar cylinder. The third gas line is not used for the present work.

We used a rf power supply with an automatic matching network and an option to connect a dc power supply in series with the rf generator to modify the dc bias of the inner electrode. To measure the etch rate of niobium, we opted for ring type samples, shown in Fig. 5. The full circle ring was attached to the inside wall of the outer electrode. The ring sample is made of $\mathrm{Nb}$ ribbon of $2.5 \mathrm{~cm}$ width. Its diameter of $7.2 \mathrm{~cm}$ corresponds to the beam tube diameter of the single cell SRF cavity. It was expected that the ring sample would exhibit a more precise rate of etching performance, as it covered a wide curved surface area, several orders of magnitude higher than previous flat sample areas.

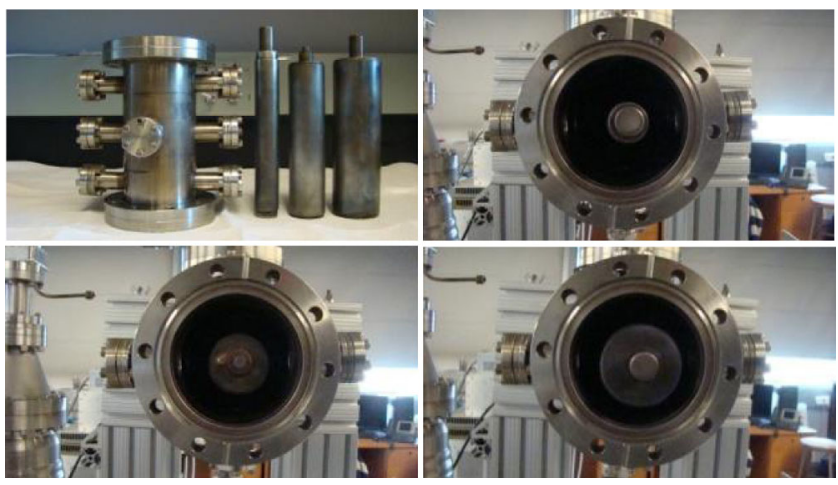

FIG. 3. Cylindrical cavity and inner electrodes with varying diameter. Clockwise: the disassembled arrangement and three end-on views of the assembled electrode configuration, starting with the lowest diameter of the electrode. 


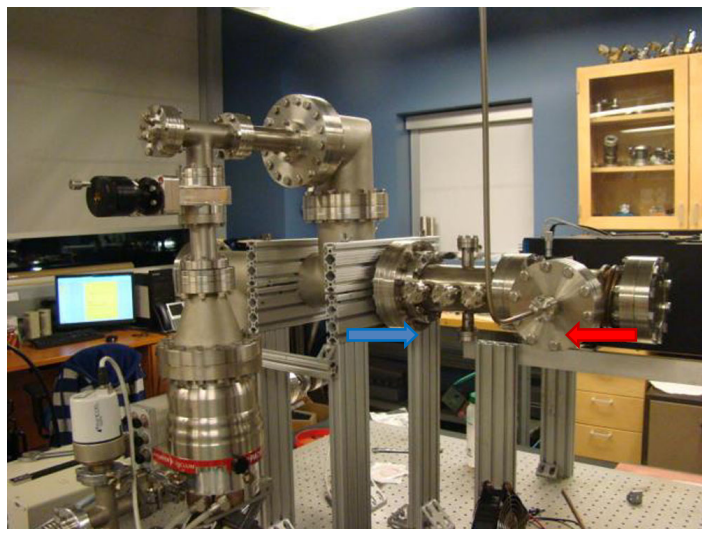

FIG. 4. Experimental setup for cylindrical plasma processing.

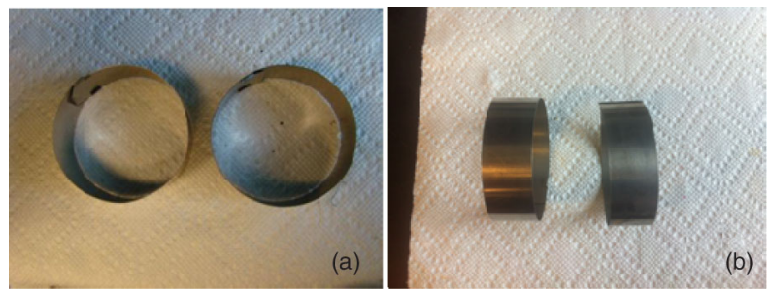

FIG. 5. Etching samples: (a) top view (b) side view.

As the plasma properties and, in turn, the surface processing effect vary substantially with frequency, pressure, chlorine concentration, temperature, and power levels inside the reactor, we have to optimize these parameters for the most efficient and uniform surface material removal from samples placed on the cavity perimeter.

\section{EXPERIMENTAL RESULTS}

Before presenting the experimental results of the processing study with the cylindrical cavity, we illustrate the normal current effect [6]. Depending on the diameter of the driven electrode there is a certain pressure (above 20 mTorr) where the plasma completely fills the cavity. When operated below the critical normal pressure the plasma is only spread over a certain section of the cavity. This effect is illustrated by observation at various pressures, as shown in Fig. 6.

Although much care was taken to retain the plasma in the full cavity, the attempt to etch a niobium sample on the outer wall without applying the positive dc bias on the driven electrode was not successful. We tried all possible pressure and power ranges in the given setup, but there was no appreciable material removal.

\section{A. Dependence of the etching rate and self-bias voltage on the diameter of the driven electrode}

As stated before, when the diameter of the driven electrode is varied, the electrode surface area ratio is

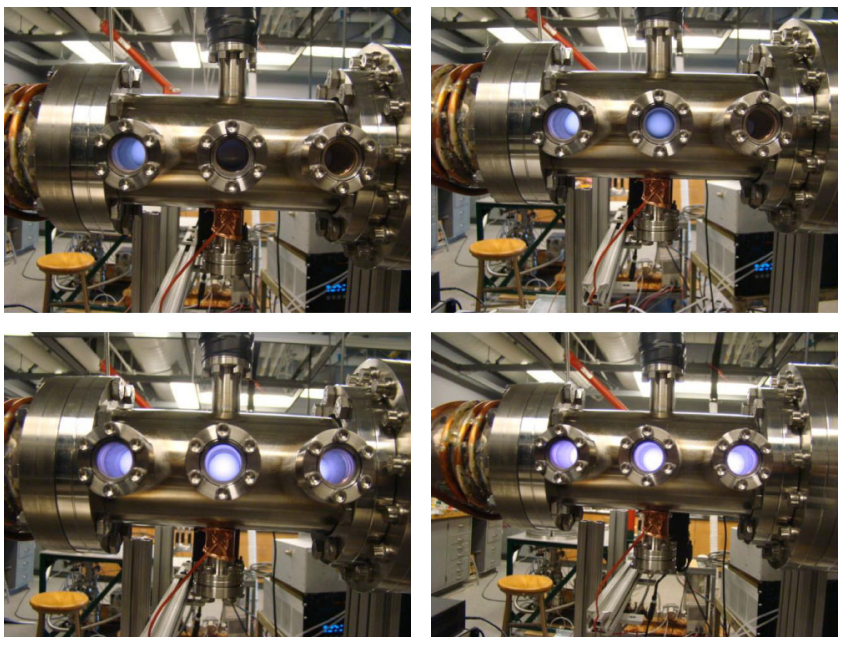

FIG. 6. Spreading of plasma inside the cylindrical cavity at different conditions: top left: lower pressure; top right: medium pressure; bottom left: higher pressure at low power; and bottom right: higher pressure and higher power.

changed and, as a consequence, the negative self-bias potential developed across the inner electrode sheath varies. We are, however, interested in the etching rate variation on the grounded (outer) electrode. Constant dc bias on the smaller diameter inner electrode does not lift the plasma potential around the grounded electrode by the same amount, due to its substantially lower surface area.

We measured the variation of the etching rate with the diameter because the etch rate also depends on the volume of produced plasma, which increases when we decrease the diameter of the inner electrode and it can affect the etch rate although the plasma potential is lower. All etch rate data were taken at constant dc bias of $+290 \mathrm{~V}$ applied on the inner electrode. The negative self-bias potential was measured at the same pressure, power, and gas concentration. The measured etching rate and self-bias potential dependence on the inner electrode diameter are given in Fig. 7.

\section{B. Dependence of etching rate on the pressure in the system}

There are three competing effects associated with the variation of pressure in the system. Increased pressure means increased concentration of radicals, which are the excited neutral atoms, molecules, and reactive ions of the chlorine. The concentration of molecules is directly proportional to the pressure, but the concentration of reactive ions depends on the rf power and the electronegativity of the discharge. Therefore, at constant rf power, a larger etch rate at higher pressure is indicative of chemical etching, and a smaller etch rate on higher pressure suggests the reactive ion etching mechanism. In addition, pressure increase leads to lower residence time and increase in collision rates among gas molecules, which leads to depletion of radicals. The increase of pressure was obtained by increasing the gas flow rate. 


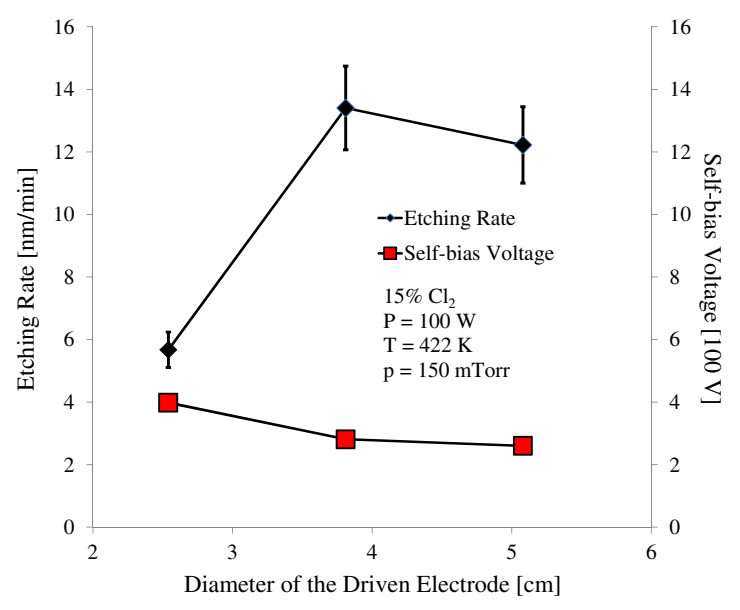

FIG. 7. Etching rate and self-bias dependence on the diameter of the driven electrode. Solid lines are visual guidelines.

The variation of etching rate with pressure at fixed electrode diameter, constant power, and constant chlorine content is given in Fig. 8. The gas mass flow rates to achieve indicated pressures are $0.25,0.40,0.55$ and $0.69 \mathrm{l} / \mathrm{m}$, respectively. All measurements were made at $\mathrm{T}=422 \mathrm{~K}$. The error in $\mathrm{Cl}_{2}$ concentration was $2 \%$, in power $3 \mathrm{~W}$, in pressure $4 \mathrm{mTorr}$, in temperature $2 \mathrm{~K}$, and in dc bias $2 \mathrm{~V}$. The etch rate is obtained by measuring the mass difference before and after the plasma exposure of the ring sample and dividing it by the area and the density of niobium and the processing time. The processing time was $100 \mathrm{~min}$ in every measurement. The removed layer thickness was $2-9 \mu \mathrm{m}$.

The calculated error in measurement of mass and area leads to an error in etch rate of $0.8 \mathrm{~nm} / \mathrm{min}$. When the error in pressure, power, chlorine concentration, temperature, and dc bias is included, we estimate the error to be about $10 \%$ in etch rate.

The results show that operating at low pressure is more favorable. The maximum etching rate measured was obtained at about 60 mTorr. It then decreased and showed saturation behavior with increasing pressure.

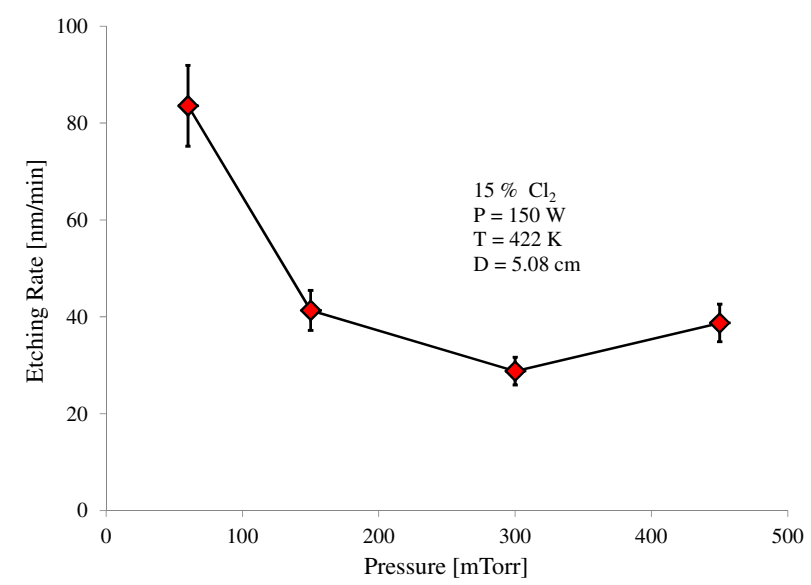

FIG. 8. Etching rate dependence on the gas pressure. Solid line is visual guideline.

\section{Dependence of etching rate on the rf power}

The variation of the etching rate with power and all other parameters kept constant is shown in Fig. 9. It follows the general property of reactive $\mathrm{rf}$ discharges that increase in power increases the amount of radicals and ions at a given pressure, which in turn increases the etching rate. In this case, the trend did not saturate due to the limited power range.

There is, however, a difference between the present case and the general trend in planar technology used in the semiconductor etching industry. In planar geometry, increasing power also increases the self-bias of the driven electrode which in turn helps in increasing the etch rate. In our case the dc bias, pressure, chlorine concentration, and temperature remain constant.

Our data indicate that there are two regimes of etching rate increase with power. Between 100 and $150 \mathrm{~W}$, the etching rate increases according to the power law with exponent of three. Above $150 \mathrm{~W}$, the increase becomes steeper. This is consistent with the transition to inductively coupled plasma reported in Refs. [13,14].

\section{Dependence of etching rate on the chlorine concentration}

In Fig. 10 we present the dependence of the average etching rate on $\mathrm{Cl}_{2}$ concentration diluted in Ar. The concentration of chlorine was changed by mixing gases from two cylinders, one $15 \% \mathrm{Cl}_{2}$ diluted in $\mathrm{Ar}$ and the other pure Ar. The lower percentages of chlorine $10 \%$ and $5 \%$ were achieved by reducing the flow of $\mathrm{Cl}_{2} / \mathrm{Ar}$ mixture to $0.261 / \mathrm{m}, 0.13 \mathrm{l} / \mathrm{m}$, respectively, and increasing the flow of pure Ar to achieve the same pressure. In this way, the gas pressure in the experiment was kept constant. Other parameters, such as rf power, dc bias, and temperature remained constant during the experiment.

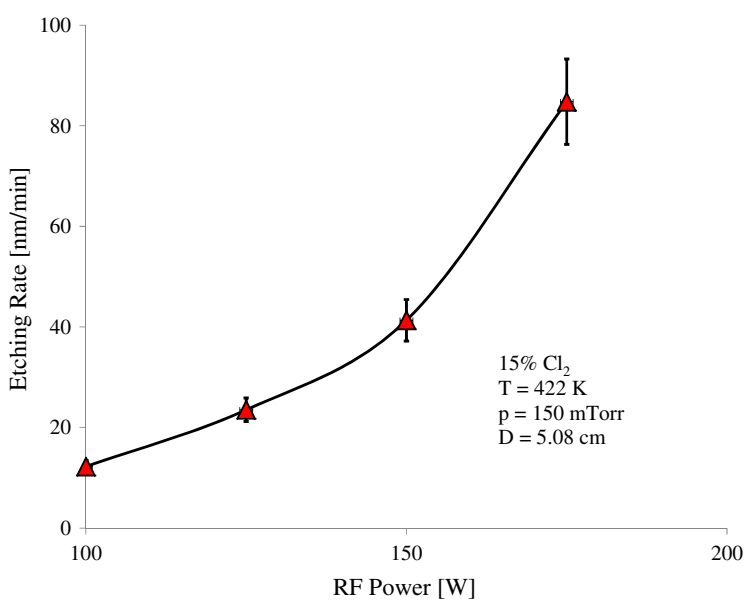

FIG. 9. Etching rate dependence on rf power. Solid line is the exponential fit. 


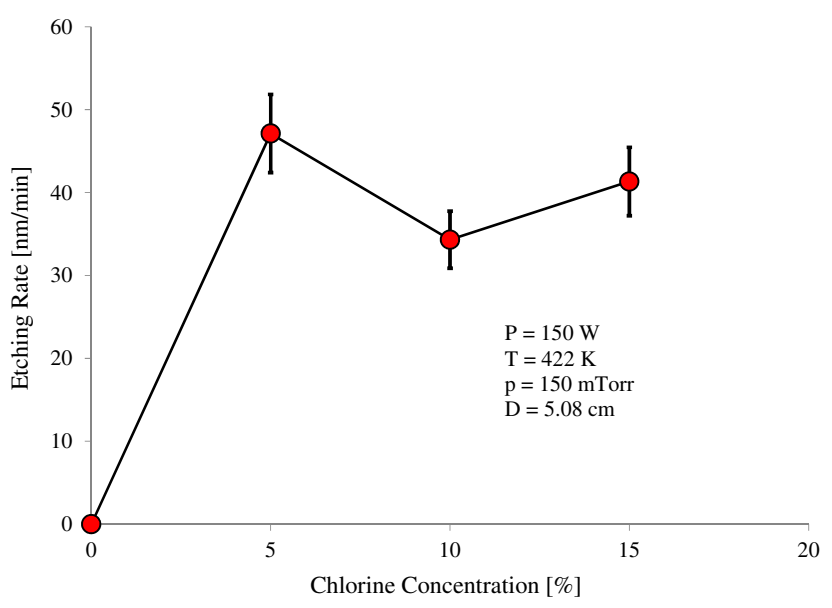

FIG. 10. Etching rate dependence on concentration of chlorine $(\% \mathrm{Vol})$ diluted in argon. Solid line is visual guideline.

There are several possible explanations for the saturation effect at relatively low chlorine concentration (see Fig. 10). First, additional chlorine may not have been consumed completely on the surface reactions, as has been observed in $\mathrm{Ar} / \mathrm{Cl}_{2}$ discharges [15]. Second, the chlorine residence time may not be long enough to enable the surface reactions. Further study will elucidate the role of these two effects. We note also that at low power, the discharge is capacitively coupled and electronegative [14], where the electron density is reduced of the expense of negatively charged chlorine ions. This saturation effect may be related to relative electron density reduction due to the increased electronegativity of the discharge. However, positive chlorine ion density, which may be important for the reactive ion etching, should not be affected by the buildup of electronegativity. Consequently, the assumed mechanism of saturation based on the increased electronegativity has to be studied in more detail.

\section{CONCLUSION}

In view of the complex technological challenges facing the development of plasma-assisted SRF surface etching, we have adopted a multistep approach to transition from flat coupons to the full scale treatment of large cavity surfaces. We have completed the intermediary step of the transition from planar to cylindrical geometry, where a curved $\mathrm{Nb}$ sample with the surface area substantially larger than flat coupons was successfully processed at a satisfactory etching rate and at a relatively modest power level.

We have measured the dependence of etching rate on the driven electrode diameter, $\mathrm{rf}$ power, gas pressure, and chlorine concentration. All measurements were performed at $422 \mathrm{~K}$. The conditions for etching are favorable at large electrode radius, where the self-bias voltage is smaller. We note that the asymmetry is reduced at a higher diameter of the driven electrode. This result also means that the sheath voltage amplitude at the grounded electrode is relatively higher, which is the more favorable condition for the reactive ion etching.

Experiments at limited rf power have shown reasonable average etching rates. The increase of the etching rate is expected to somewhat slow down at higher power, but will certainly provide satisfactory etching rates when combined with temperature and pressure adjustments.

Experiments on gas pressure variation indicate favorable conditions at low pressure, as observed in a number of works in planar technology. It seems that the increase of chlorine concentration above 5\% does not provide any substantial benefit to the process.

In the final remark, we have demonstrated a relatively robust etching of a cylindrical $\mathrm{Nb}$ sample of diameter comparable to a cylindrical cavity. This result presents a successful intermediary step in the transition from flat coupons etching to the cylindrical cavity wall processing.

\section{ACKNOWLEDGMENTS}

This work is supported by the Office of High Energy Physics, Office of Science, Department of Energy under Grant No. DE-SC0007879. J. U. is supported by the Thomas Jefferson National Accelerator Facility, Accelerator Division.

[1] P. Kneisel, Nucl. Instrum. Methods 557, 250 (2006).

[2] M. Rašković, J. Upadhyay, L. Vušković, S. Popović, A.-M. Valente-Feliciano, and L. Phillips, Phys. Rev. ST Accel. Beams 13, 112001 (2010).

[3] M. Rašković, J. Upadhyay, L. Vušković, S. Popović, A.-M. Valente-Feliciano, and L. Phillips, J. Vac. Sci. Technol. A 27, 301 (2009).

[4] J. Upadhyay, Do Im, S. Popović, L. Vusković, A.-M. Valente-Feliciano, and L. Phillips, Proceedings of SRF 2013, Paris, France, 2013, p. 580.

[5] Yu. P. Raizer, M. N. Shneider, and N. A. Yatsenko, RadioFrequency Capacitive Discharges (CRC Press, Berlin, 1995).

[6] M. A. Lieberman and A. J. Lichtenberg, Principles of Plasma Discharges and Material Processing (John Wiley \& Sons, New York, 1994).

[7] H. R. Koenig and L. I. Maissel, IBM J. Res. Dev. 14, 168 (1970).

[8] J. W. Coburn and E. Kay, J. Appl. Phys. 43, 4965 (1972).

[9] J. L. Vossen, J. Electrochem. Soc. 126, 319 (1979).

[10] C. M. Horwitz, J. Vac. Sci. Technol. A 1, 60 (1983).

[11] K. Kohler, J. W. Coburn, D. E. Horne, E. Kay, and J. H. Keller, J. Appl. Phys. 57, 59 (1985).

[12] M. A. Lieberman and S. E. Savas, J. Vac. Sci. Technol. A 8, 1632 (1990).

[13] M.-M. Chen and Y. H. Lee, J. Electrochem. Soc. 131, 2118 (1984).

[14] M. V. Malyshev and V. M. Donnely, J. Appl. Phys. 90, 1130 (2001).

[15] C. J. Mogab, J. Electrochem. Soc. 124, 1262 (1977). 